\title{
Atrial Fibrillation - An Orchestra of Classic and Modern Risk Factors
}

\begin{abstract}
Alkora Ioana Balan, Alina Scridon*
University of Medicine and Pharmacy Science and Technology of Targu Mures, Romania

Over the past years, prevention and control of risk factors has begun to play an important role in the management of patients prone to develop atrial fibrillation (AF). A considerable number of risk factors that contribute to the creation of a predisposing substrate for AF has been identified over the years. Although certain AF risk factors such as age, gender, genetic predisposition, or race are unmodifiable, controlling modifiable risk factors may represent an invaluable tool in the management of AF patients. In the recent decades, numerous studies have evaluated the mechanisms linking different risk factors to AF, but the exact degree of atrial remodeling induced by each factor remains unknown. Elucidating these mechanisms is essential for initiating personalized therapies in patients prone to develop AF. The present review aims to provide an overview of the most relevant modifiable risk factors involved in AF occurrence, with a focus on the mechanisms by which these factors lead to $A F$ initiation and perpetuation.
\end{abstract}

Keywords: atrial fibrillation, epidemiology, mechanisms, remodeling, risk factors

Received 30 May 2019 / Accepted 4 August 2019

\section{Introduction}

Atrial fibrillation (AF), the most common sustained cardiac arrhythmia, can represent both a cause and a consequence of numerous cardiac and non-cardiac diseases. The prevalence of $\mathrm{AF}$ is steadily increasing with the aging of the population and the presence of the arrhythmia is associated with a substantial number of risk factors and clinical outcomes [1]. Numerous AF risk factors have been identified, with a variable degree of reversibility (Table I).

Table I. Atrial fibrillation risk factors

\begin{tabular}{ccc}
\hline $\begin{array}{c}\text { Unmodifiable risk } \\
\text { factors }\end{array}$ & $\begin{array}{c}\text { Modifiable risk } \\
\text { factors }\end{array}$ & $\begin{array}{c}\text { Partially modifiable risk } \\
\text { factors }\end{array}$ \\
\hline Age & Obesity & Arterial hypertension \\
Gender & Sedentary lifestyle & Diabetes mellitus \\
Genetic background & Physical activity & Heart failure \\
Race & Smoking & Ischemic heart disease \\
& Alcohol consump- & Chronic kidney disease \\
& tion & Obstructive sleep apnea \\
& & Chronic obstructive pulmo- \\
& & nary disease
\end{tabular}

Moreover, a two-way relationship appears to exist between AF and many of its risk factors. Factors such as arterial hypertension, aging, heart failure, or ischemic heart disease have long been recognized as major AF risk factors [1]. Another series of factors such as diabetes mellitus, obesity, sedentary lifestyle, obstructive sleep apnea, chronic kidney disease (CKD), and chronic obstructive pulmonary disease (COPD) have been added to this list of AF risk factors more recently $[1,2]$. Recent studies have also described a relationship between behavioral and environmental fac-

* Correspondence to: Alina Scridon

E-mail: alinascridon@gmail.com tors, including smoking, chronic alcohol consumption, and air pollution, and AF [1]. Atrial fibrillation pathophysiology has been linked to electrical, structural and autonomic abnormalities, and all risk factors involved in AF pathogenesis have been shown to induce one or several of these abnormalities (Figure 1).

The present review aims to provide an overview of the classic and more modern modifiable AF risk factors and to discuss the main mechanisms through which these factors promote the initiation and/or maintenance of nonvalvular AF.

\section{Arterial Hypertension}

In the Framingham Heart Study cohort, high blood pressure was associated with a 1.8 -fold increased risk of developing $A F$, and, given the increased prevalence of high blood pressure among the study patients, hypertension was responsible for $14 \%$ of all AF cases [1]. Particularly, a positive correlation was found between AF and systolic blood pressure [2].

In addition to inducing ventricular hypertrophy and atrial dilation, arterial hypertension has also been shown to cause hypertrophy at the atrial level, which could contribute to the increased risk of AF in this setting [3]. Reduced left atrial function and progressive atrial fibrosis associated with inflammatory infiltrates have also been reported [3]. Overactivation of the renin-angiotensin-aldosterone system (RAAS) has been incriminated as the most relevant mechanism involved in this hypertension-induced atrial proarrhythmic remodeling $[4,5]$. Increased expression of the angiotensin-converting enzyme and abnormal angiotensin II type 1 and type 2 receptors expression have been reported in this setting [4,5]. Meanwhile, candesartan, an angiotensin II type 1 receptor blocker, was shown to efficiently block angiotensin II-induced collagen synthesis 


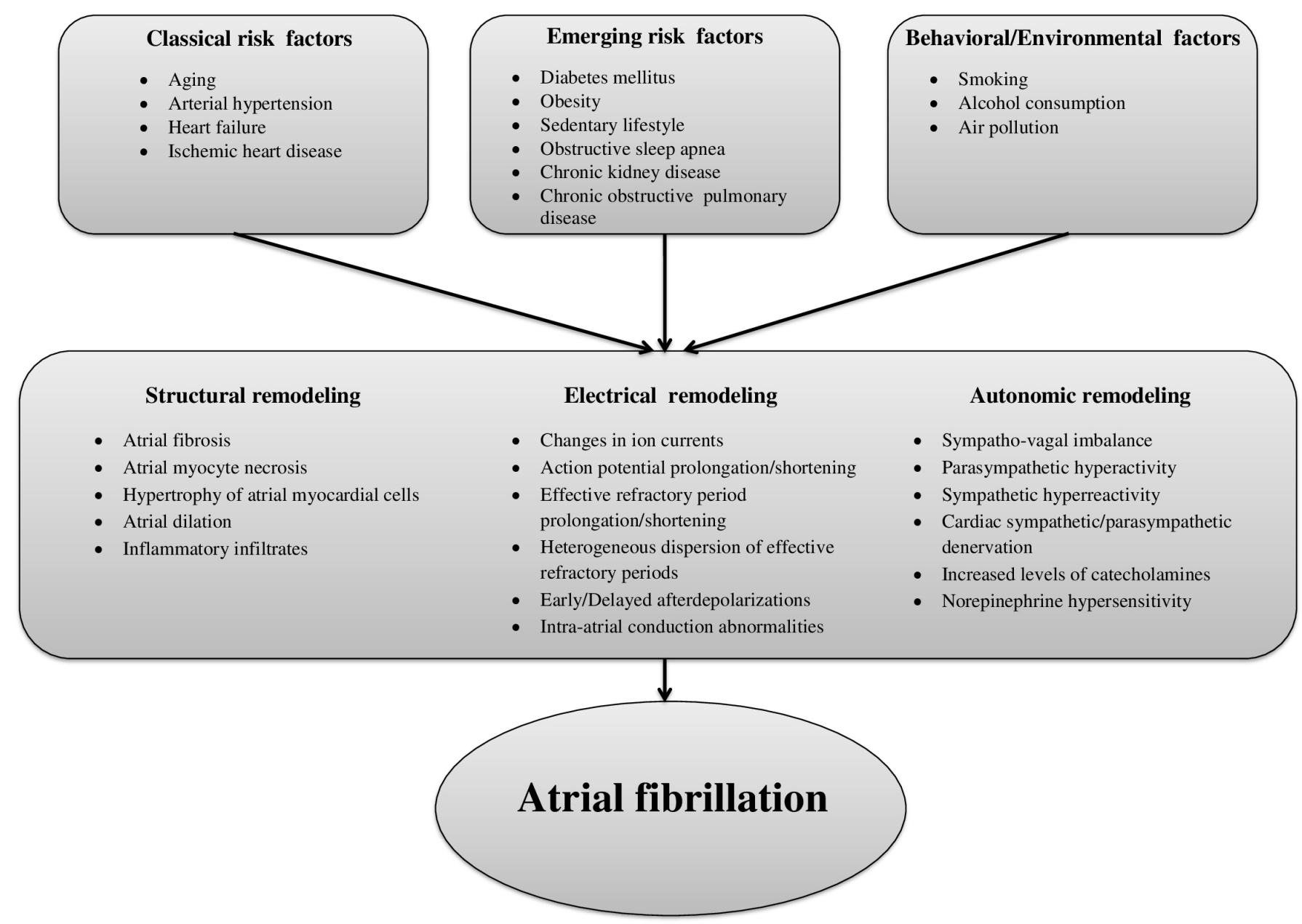

Fig. 1. Schematic representation of the mechanisms linking atrial fibrillation (AF) to its major risk factors. The figure depicts the effects of $\mathrm{AF}$ risk factors on the atria, which will ultimately lead to $\mathrm{AF}$ initiation and/or maintenance.

and to reduce atrial fibrosis in a hypertensive rat model [6]. Decreased intra-atrial conduction velocity, together with increased heterogeneity and increased duration of atrial effective refractory periods (ERPs), has also been reported in the presence of arterial hypertension [3]. Sympatho-vagal imbalance has been identified more recently as a contributor to AF occurrence in this setting. In spontaneously hypertensive rats, reduced sympathetic tone and relative vagal hyperactivity have been shown to precede and favor AF occurrence [7]. In that study, increased sympathetic tone induced by emotional stress restored the autonomic balance and decreased the number of arrhythmic events, whereas parasympathetic stimulation significantly increased atrial arrhythmic burden and triggered AF [7].

\section{Heart failure}

Whereas heart failure is seen as a major risk factor for AF, $\mathrm{AF}$ can also be seen as a contributing factor to heart failure $[1,8]$. In a cohort study, $26 \%$ of the patients diagnosed with heart failure developed AF over a mean follow-up of 4.2 years, whereas the incidence of heart failure among $\mathrm{AF}$ patients was 33 per 1,000 person-years [8].

In an experimental study in dogs, heart failure was associated with atrial fibrosis, whereas treatment with pirfe- nidone, an anti-fibrotic agent, attenuated these structural changes and significantly decreased AF susceptibility [9]. Changes in intracellular calcium, characterized by increased calcium transient amplitude and sarcoplasmic reticulum calcium overload, also appear to provide an AFsusceptible substrate in this setting [10]. Prolonged ERP and action potential duration, predisposing to early afterdepolarizations, have been reported in dogs with heart failure $[9,10]$, probably due to decreased activities of the transient outward $\mathrm{K}^{+}$current $\left(I_{t o}\right)$ and of the slow delayed rectifier current $\left(I_{K S}\right)$ [11]. Increased activity of the $\mathrm{Na}^{+} /$ $\mathrm{Ca}^{2+}$ exchanger has also been reported in those dogs, favoring delayed afterdepolarizations and AF [11]. However, neurohormonal activation, characterized by increased release of catecholamines and angiotensin II, appears to be the main mechanism linking heart failure to $\operatorname{AF}[12,13]$, whereas angiotensin II receptors blockers and angiotensinconverting enzyme inhibitors have been shown to efficiently prevent AF occurrence in this setting [14].

\section{Ischemic heart disease}

Ischemic heart disease and AF often coexist in the same patient and can potentiate one another [15]. In the Framingham Heart study cohort, one fourth of men with coronary 
heart disease developed chronic AF [16]. The risk to develop transient $\mathrm{AF}$ was four times higher in women with than in those without coronary heart disease, although there was no significant association between coronary heart disease and chronic AF [16]. In the same study, a 3-fold and a 9-fold increase in the risk of developing transient $\mathrm{AF}$ was also reported in men and women with a previous acute coronary syndrome, respectively [16]. Inflammation, a key player in the pathogenesis of coronary artery disease, has been shown to promote AF via structural, electrical, and/ or autonomic remodeling [15]. In a study on rabbit isolated left atria, hypoxia induced electrophysiological changes characterized by increased ERP and decreased conduction velocity, increasing vulnerability to reentry [17]. In dogs, atrial ischemia led to local conduction slowing, favoring AF maintenance [18]. Finally, ischemic heart disease leads to myocardial dysfunction and heart failure, which are independent risk factors for AF $[1,8]$.

\section{Diabetes mellitus}

Numerous studies have reported an increased incidence of $\mathrm{AF}$ in diabetic patients and a linear relationship has been observed between both the duration of diabetes and HbA1c levels and AF risk [19]. However, the diabetes mellitus-AF relationship is far from clear. Although a large amount of data indicates diabetes mellitus as an independent risk factor for $\mathrm{AF}$, to date, there is no definitive proof that diabetes per se is sufficient to ensure AF occurrence [19].

Nevertheless, in female patients, impaired glucose tolerance was associated with increased left ventricular mass [20], a known AF risk factor. The systemic inflammatory syndrome commonly encountered in diabetic patients [19] has also been placed amongst the mechanisms linking diabetes to AF. Increased interleukin- 6 and C-reactive protein (CRP) levels have been identified in diabetic patients [19], whereas inflammation is known to precipitate AF. Increased fibrosis was also observed in rats with type II diabetes compared to controls, leading to intra-atrial conduction abnormalities [21]. Electrical remodeling of the atria has been reported in diabetic rats, as well as in patients with abnormal glucose metabolism, which displayed intra-atrial conduction abnormalities and decreased atrial voltage [21,22]. Finally, in diabetic rats, inducibility of sustained AF was associated with heterogeneous cardiac sympathetic denervation and homogenous cardiac parasympathetic denervation, and both sympathetic and parasympathetic stimulation have been shown to increase AF occurrence [23].

\section{Obesity}

Numerous population-based studies have associated obesity with an increased risk of AF [2,24]. A 4\% increase in the risk of $\mathrm{AF}$ was observed for each unit added to the body mass index [24], whereas in patients with paroxysmal or persistent AF, weight loss had a dose-dependent effect on maintaining sinus rhythm and reducing the risk of recurrent $\mathrm{AF}[25]$.
In sheep, obesity was associated with left atrial enlargement, atrial fibrosis, and pericardial lipid deposits, contributing to AF initiation and maintenance [26]. Direct release of inflammatory cytokines by the epicardial fat has also been incriminated in AF occurrence in obese patients, via pericardiac ganglionated plexuses stimulation, parasympathetic-induced ERP shortening and intra-atrial conduction slowing, and sympathetic-induced increase in calcium transient in the atria and the pulmonary veins [15]. In patients undergoing pulmonary vein isolation, obesity has been associated with shorter ERP [27], although inhomogeneous action potential prolongation has also been reported as a potential AF predisposing factor in obese patients [28].

\section{Obstructive sleep apnea}

Extensive evidence has associated sleep apnea with an increased risk of developing AF [29]. In addition, sleep apnea and $\mathrm{AF}$ share a number of risk factors such as hypertension, obesity, diabetes mellitus, and coronary artery disease [2]. The Sleep Heart Study, which compared patients without sleep-disordered breathing with patients with obstructive sleep apnea, reported a 4-fold higher prevalence of AF in the latter [29], whereas continuous positive airway pressure treatment decreased AF risk in this setting [2].

Repeated forced inspiration leading to decreased intrathoracic pressure favors atrial filling and increases intraatrial pressure, leading to atrial enlargement and AF [30]. Hypoxia and hypercapnia produced during repeated apnea episodes activate the chemoreceptor reflex, induce autonomic dysfunction, and increase the blood pressure, thus favoring AF occurrence [31]. Inflammation, as evidenced by increased oxidative stress and elevated CRP levels, also appears to promote atrial remodeling and $\mathrm{AF}$ in these patients [32]. In addition, the hypercapnia that occurs during apnea episodes has been associated with a uniform increase in ERP and with slowed intra-atrial conduction, mediated by chemoreceptor-induced sympathetic activation [33], while application of a negative pressure during tracheal occlusion, associated with vagal activation, induced a significant decrease in ERP [34].

\section{Sedentary lifestyle and physical activity}

There is a nonlinear relationship between physical activity and AF; both sedentary lifestyle and intense physical activity have been associated with increased AF risk [35,36]. In a retrospective cohort study assessing the impact of cardiopulmonary fitness on $\mathrm{AF}$ risk, a $7 \%$ decrease in $\mathrm{AF}$ risk was observed for each metabolic equivalent added during treadmill testing [35]. At the opposite pole, a 5-fold higher risk of $\mathrm{AF}$ has been reported in athletes, compared to the general population [36].

The association between reduced physical activity and AF risk factors including diabetes, obesity, and hypertension, is probably one of the main mechanisms by which sedentary lifestyle increases AF risk [37]. Adiposity-asso- 
ciated inflammation has been observed in patients with physical inactivity, further contributing to atrial proarrhythmic remodeling [38]. The increased sympathetic tone generally present in these people could also contribute to AF by promoting early and/or delayed afterdepolarizations [39].

Meanwhile, elevated levels of fibrosis biomarkers have been reported in athletes [40] and atrial fibrosis has been highlighted by histological examination in physically trained rats [41]. Atrial dilation, induced by intense physical training as an adaptation to the increased cardiac output, also contributes to AF susceptibility in this setting [41]. Finally, autonomic imbalance has also been incriminated in AF occurrence in trained athletes. Parasympathetic-induced bradycardia, commonly seen in athletes, has been associated with ERP shortening, increased likelihood of reentry, and higher risk of AF occurrence [42].

\section{Chronic kidney disease}

Chronic kidney disease is recognized as a strong predictor of cardiovascular events, including AF [43]. The association between $\mathrm{CKD}$ and new-onset $\mathrm{AF}$ has been reported in several population-based studies and an increased risk of developing AF has been seen in patients with glomerular filtration rate below $60 \mathrm{ml} / \mathrm{min} / 1.73 \mathrm{~m}^{2}$ [43].

In patients with CKD, the primary role in atrial proarrhythmic remodeling has been attributed to RAAS activation [14]. Low-grade inflammation and increased oxidative stress, commonly seen in patients with CKD, may also play an important role in this regard [44]. Meanwhile, in a model of renal failure in rats, administration of antioxidant agents significantly reduced AF inducibility [44]. Increased levels of catecholamines and enhanced norepinephrine hypersensitivity, typically seen in CKD patients, may also contribute to increased AF susceptibility in this setting [45].

\section{Chronic obstructive pulmonary disease}

Numerous studies have linked the presence and the exacerbations of COPD with an increased risk of AF $[46,47]$, whereas forced expiratory volume was negatively correlated with AF occurrence [48]. The role of hypoxia as a possible mechanism for AF promotion in the setting of COPD remains controversial. While some studies have failed to demonstrate a direct effect of hypoxia on the electrophysiological properties of atria [33], others described a hypoxemia-induced inhomogeneous conduction of premature wavefronts, shortened wavelength [18], and sympathetic overactivation [31]. Meanwhile, a consensus seems to have been reached regarding the impact of hypercapnia on the electrophysiological properties of the atria. Hypercapniainduced atrial conduction slowing and ERP prolongation appear to create a substrate for AF, even after carbon dioxide returns to normal values [33]. In COPD patients, AF occurrence has been linked to prolonged atrial depolarization and electromechanical delay [49]. Increased oxidative stress and systemic inflammation have also been observed in COPD patients [50]. Increased right atrial volume secondary to right ventricular systolic dysfunction and pulmonary arterial hypertension could further increase the AF risk in this population [46].

\section{Smoking}

Both current and former smokers seem to have increased risk of developing $\mathrm{AF}$ and a dose-response relationship appears to characterize this association [51]. Indirectly, smoking predisposes to myocardial infarction, heart failure, and COPD, all of which are independent risk factors for $\mathrm{AF}$. However, the smoking-AF association appears to extend far beyond these smoking-related conditions. One of the main mechanisms by which tobacco smoking induces AF appears to be myocardial ischaemia, mainly due to decreased blood oxygen carrying capacity, coronary vasoconstriction, and accelerated atherosclerosis [52]. Interstitial fibrosis that occurs in these patients has been attributed to increased transforming growth factor $\beta$ (TGF- $\beta$ ) and type II TGF- $\beta$ receptors levels [53]. Nicotine-induced autonomic dysfunction, characterized by down-regulation of beta-adrenergic receptors and increased release of catecholamines, has also been incriminated in AF development [54]. Although the effect of nicotine on atrial ion channels has not been studied, at the ventricular level, nicotine-induced blockade of the inward rectifier potassium channels could promote ectopic and triggered activity and induce arrhythmias [55].

\section{Alcohol consumption}

The association between alcohol consumption and AF appears to be dose- and gender-dependent [56]. Whereas low alcohol intake does not seem to be associated with AF, moderate alcohol consumption has only been associated with $\mathrm{AF}$ in males, and a gender-independent association has been observed between high doses of alcohol and AF occurrence [56]. An association between excessive acute alcohol intake and cardiac arrhythmias, known as the "Holiday heart syndrome", has also been described [56]. One of the mechanisms explaining the alcohol intake-AF association relies on the proarrhythmic effect of acetaldehyde, the primary metabolite of alcohol, on the Purkinje fibers [57]. To date, the effect of alcohol consumption on atrial structural remodeling has not been adequately studied. Qiao et al. recently reported an association between alcohol consumption and the presence of low-voltage areas, speculating a relationship between alcohol consumption and atrial fibrosis [58]. Meanwhile, alcohol-induced electrical remodeling has been studied more extensively. Prolonged (120-h) exposure of rabbits to high intravenous alcohol infusion resulted in a significant decrease in $I_{\text {Ca-L }}$ and sodium current $\left(I_{\mathrm{Na}}\right)$ density [59]. Decreased ERP and prolonged intra-atrial conduction have also been reported following alcohol ingestion in patients undergoing electrophysiological study [60]. Changes in the duration of the $\mathrm{P}$ 
wave and of the PR interval further support proarrhythmic intra-atrial conduction prolongation following alcohol consumption [61]. Finally, autonomic dysfunction may also contribute to alcohol intake-related AF. In healthy individuals, acute alcohol ingestion has been associated with a decrease in short-term heart rate variability (HRV) and with an increase in low-to-high-frequency (LF/HF) HRV components ratio, suggesting that sympathetic overactivity may be involved in binge drinking-related AF [62].

\section{Air pollution}

Epidemiological evidence, mostly derived from studies in patients with implanted cardioverter defibrillators, associates air pollution with an elevated risk of AF [63], mainly via pollution-induced inflammation [64]. In addition, increased levels of pollutants, particularly carbon monoxide and carbon black, have been associated with HRV reduction [65]. Carbonaceous particles have also been shown to increase the LF/HF ratio, demonstrating an increased sympathetic activity in this setting [65]. Increased complexity of the $\mathrm{P}$ wave and $\mathrm{PR}$ prolongation as a result of acute exposure to particulate matter $<2.5 \mu \mathrm{m}$ in aerodynamic diameter have also been linked to increased AF vulnerability, although the exact mechanisms by which these changes occur remain to date unknown [66]. Finally, air pollutants can also favor AF occurrence by inducing COPD and heart failure exacerbations and/or by aggravating coronary artery disease [63].

\section{Clinical Implications}

Over the time, clinical and experimental studies have identified a variety of AF risk factors and provided insights into their mechanistic links to AF genesis. Elucidating the mechanisms by which various risk factors lead to AF has an indisputable role in identifying new prevention methods. While AF risk factors such as age are unmodifiable (Table I), correction of the numerous modifiable AF risk factors could considerably reduce the AF burden. Indeed, studies have shown that lifestyle changes and/or treatment of clinical conditions commonly associated with AF lead to a significant decrease in the risk of new-onset AF [67]. Although numerous AF risk factors have been identified, clinical risk scores have a limited capacity to predict AF, highlighting once more the complexity of this arrhythmia. Patients often display multiple AF risk factors, and AF mechanisms are probably different from one patient to another. All this suggests that patients at risk of AF probably require a personalized approach and that risk factors management requires an integrated multidisciplinary strategy.

\section{Conclusion}

In their vast majority, AF risk factors play an important role in inducing proarrhythmic atrial structural, electrical, and/or autonomic remodeling, but they also often contribute to the development of other risk factors, which will in- dependently contribute, at their turn, to AF initiation and maintenance. Elucidating the mechanisms by which these factors contribute to AF initiation and perpetuation is expected to provide a basis for new antiarrhythmic strategies. In the meantime, adequate management of modifiable $\mathrm{AF}$ risk factors could represent a valuable tool for reducing the AF burden in the general population.

\section{Authors'contribution}

Alkora Ioana Balan (Conceptualization; Data curation; Formal analysis; Investigation; Methodology; Writing original draft)

Alina Scridon (Conceptualization; Data curation; Formal analysis; Funding acquisition; Investigation; Methodology; Project administration; Resources; Supervision; Validation; Visualization; Writing - review \& editing)

\section{Acknowledgments}

This work was supported by a grant of the Ministery of Research and Innovation, CNCS-UEFISCDI, project number PN-III-P1-1.1-TE-2016-0382, within PNCDI III.

\section{Conflict of interest}

None to declare.

\section{References}

1. Benjamin EJ, Levy D, Vaziri SM, D’Agostino RB, Belanger AJ, Wolf PA. Independent risk factors for atrial fibrillation in a population-based cohort. The Framingham Heart Study. JAMA. 1994;271:840-844.

2. Brandes A, Smit MD, Nguyen BO, Rienstra M, Van Gelder IC. Risk factor management in atrial fibrillation. Arrhythm Electrophysiol Rev. 2018;7:118-127.

3. Lau DH, Mackenzie L, Kelly DJ, et al. Short-term hypertension is associated with the development of atrial fibrillation substrate: a study in an ovine hypertensive model. Heart Rhythm. 2010;7:396-404.

4. Goette A, Staack T, Rocken C, et al. Increased expression of extracellular signal-regulated kinase and angiotensin-converting enzyme in human atria during atrial fibrillation. J Am Coll Cardiol. 2000;35:1669-1677.

5. Goette A, Arndt M, Rocken C, et al. Regulation of angiotensin II receptor subtypes during atrial fibrillation in humans. Circulation. 2000;101:26782681.

6. Okazaki $\mathrm{H}$, Minamino $\mathrm{T}$, Tsukamoto $\mathrm{O}$, et al. Angiotensin ॥ type 1 receptor blocker prevents atrial structural remodeling in rats with hypertension induced by chronic nitric oxide inhibition. Hypertens Res. 2006;29:277-284

7. Scridon A, Gallet C, Arisha MM, et al. Unprovoked atrial tachyarrhythmias in aging spontaneously hypertensive rats: the role of the autonomic nervous system. Am J Physiol Heart Circ Physiol. 2012;303:H386-H392.

8. Wang TJ, Larson MG, Levy D, et al. Temporal relations of atrial fibrillation and congestive heart failure and their joint influence on mortality: the Framingham Heart Study. Circulation. 2003;107:2920-2925.

9. Lee KW, Everett TH 4th, Rahmutula D, et al. Pirfenidone prevents the development of a vulnerable substrate for atrial fibrillation in a canine model of heart failure. Circulation. 2006;114:1703-1712.

10. Yeh $Y H$, Wakili R, Qi XY, et al. Calcium-handling abnormalities underlying atrial arrhythmogenesis and contractile dysfunction in dogs with congestive heart failure. Circ Arrhythm Electrophysiol. 2008;1:93-102.

11. Li D, Melnyk P, Feng J, et al. Effects of experimental heart failure on atrial cellular and ionic electrophysiology. Circulation. 2000;101:2631-2638.

12. Cardin S, Li D, Thorin-Trescases N, Leung TK, Thorin E, Nattel S. Evolution of the atrial fibrillation substrate in experimental congestive heart failure: angiotensin-dependent and independent pathways. Cardiovasc Res. 2003;60:315-325.

13. Bettoni M, Zimmermann M. Autonomic tone variations before the onset of paroxysmal atrial fibrillation. Circulation. 2002;105:2753-2759.

14. Nakashima $H$, Kumagai $K$, Urata $H$, Gondo N, Ideishi M, Arakawa K. Angiotensin II antagonist prevents electrical remodeling in atrial 
fibrillation. Circulation. 2000; 101:2612-2617.

15. Scridon A, Dobreanu D, Chevalier P, Șerban RC. Inflammation, a link between obesity and atrial fibrillation. Inflamm Res. 2015;64:383-393.

16. Kannel WB, Abbott RD, Savage DD, McNamara PM. Coronary heart disease and atrial fibrillation: the Framingham Study. Am Heart J. 1983;106:389-396.

17. Lammers WJ, Kirchhof C, Bonke Fl, Allessie MA. Vulnerability of rabbit atrium to reentry by hypoxia. Role of inhomogeneity in conduction and wavelength. Am J Physiol. 1992;262:H47-H55.

18. Sinno H, Derakhchan K, Libersan D, Merhi $Y$, Leung TK, Nattel S. Atrial ischemia promotes atrial fibrillation in dogs. Circulation. 2003;107:19301936.

19. Şerban RC, Scridon A. Data Linking diabetes mellitus and atrial fibrillationhow strong is the evidence? From epidemiology and pathophysiology to therapeutic implications. Can J Cardiol. 2018;34:1492-1502.

20. Rutter MK, Parise H, Benjamin EJ, et al. Impact of glucose intolerance and insulin resistance on cardiac structure and function: sex-related differences in the Framingham Heart Study. Circulation. 2003;107:448454

21. Kato T, Yamashita T, Sekiguchi A, et al. What are arrhythmogenic substrates in diabetic rat atria? J Cardiovasc Electrophysiol. 2006;17:890-894.

22. Chao TF, Suenari K, Chang SL, et al. Atrial substrate properties and outcome of catheter ablation in patients with paroxysmal atrial fibrillation associated with diabetes mellitus or impaired fasting glucose. Am J Cardiol. 2010;106:1615-1620

23. Otake H, Suzuki H, Honda T, Maruyama Y. Influences of autonomic nervous system on atrial arrhythmogenic substrates and the incidence of atrial fibrillation in diabetic heart. Int Heart J. 2009;50:627-641.

24. Wang TJ, Parise H, Levy D, et al. Obesity and the risk of new-onset atrial fibrillation. JAMA. 2004;292:2471-2477.

25. Pathak RK, Middeldorp ME, Meredith M, et al. Long-term effect of goaldirected weight management in an atrial fibrillation cohort: A long-term follow-up study (LEGACY). J Am Coll Cardiol. 2015;65:2159-2169.

26. Abed HS, Samuel CS, Lau DH, et al. Obesity results in progressive atrial structural and electrical remodeling: implications for atrial fibrillation. Heart Rhythm. 2013;10:90-100.

27. Munger TM, Dong YX, Masaki M, et al. Electrophysiological and hemodynamic characteristics associated with obesity in patients with atrial fibrillation. J Am Coll Cardiol. 2012;60:851-860.

28. Lin YK, Chen YC, Chen JH, Chen SA, Chen YJ. Adipocytes modulate the electrophysiology of atrial myocytes: implications in obesity-induced atrial fibrillation. Basic Res Cardiol. 2012;107:293-303.

29. Mehra R, Benjamin EJ, Shahar E, et al. Association of nocturnal arrhythmias with sleep disordered breathing: the Sleep Heart Health Study. Am J Respir Crit Care Med. 2006;173:910-916.

30. Orban M, Bruce CJ, Pressman GS, et al. Dynamic changes of left ventricular performance and left atrial volume induced by the mueller maneuver in healthy young adults and implications for obstructive sleep apnea, atrial fibrillation, and heart failure. Am J Cardiol. 2008;102:15571561.

31. Somers VK, Dyken ME, Clary MP, Abboud FM. Sympathetic neural mechanisms in obstructive sleep apnea. J Clin Invest. 1995;96:18971904.

32. Hatipoğlu U, Rubinstein I. Inflammation and obstructive sleep apnea syndrome pathogenesis: a working hypothesis. Respiration. 2003;70:665-671.

33. Stevenson $\mathbb{H}$, Roberts-Thomson KC, Kistler PM, et al. Atrial electrophysiology is altered by acute hypercapnia but not hypoxemia: implications for promotion of atrial fibrillation in pulmonary disease and sleep apnea. Heart Rhythm. 2010;7:1263-1270.

34. Linz D, Schotten U, Neuberger HR, Böhm M, Wirth K. Negative tracheal pressure during obstructive respiratory events promotes atrial fibrillation by vagal activation. Heart Rhythm. 2011;8:1436-1443.

35. Qureshi WT, Alirhayim Z, Blaha MJ, et al. Cardiorespiratory fitness and risk of incident atrial fibrillation: results from the Henry Ford Exercise Testing (FIT) Project. Circulation. 2015;131:1827-1834.

36. Abdulla J, Nielsen JR. Is the risk of atrial fibrillation higher in athletes than in the general population? A systematic review and meta-analysis. Europace. 2009;11:1156-1159.

37. Thorp AA, Owen N, Neuhaus M, Dunstan DW. Sedentary behaviors and subsequent health outcomes in adults a systematic review of longitudinal studies, 1996-2011. Am J Prev Med. 2011;41:207-215.

38. Allison MA, Jensky NE, Marshall SJ, Bertoni AG, Cushman M. Sedentary behavior and adiposity-associated inflammation: the Multi-Ethnic Study of Atherosclerosis. Am J Prev Med. 2012;42:8-13.
39. Mueller PJ. Exercise training and sympathetic nervous system activity: evidence for physical activity dependent neural plasticity. Clin Exp Pharmacol Physiol. 2007;34:377-384.

40. Lindsay MM, Dunn FG. Biochemical evidence of myocardial fibrosis in veteran endurance athletes. Br J Sports Med. 2007;41:447-452.

41. Guasch E, Benito B, Qi X, et al. Atrial fibrillation promotion by endurance exercise: demonstration and mechanistic exploration in an animal model. J Am Coll Cardiol. 2013;62:68-77.

42. Mont L, Elosua R, Brugada J. Endurance sport practice as a risk factor for atrial fibrillation and atrial flutter. Europace. 2009;11:11-17.

43. Shang W, Li L, Huang S, et al. Chronic kidney disease and the risk of new-onset atrial fibrillation: a meta-analysis of prospective cohort studies. PLoS One. 2016;11:e0155581.

44. Fukunaga N, Takahashi N, Hagiwara S, et al. Establishment of a model of atrial fibrillation associated with chronic kidney disease in rats and the role of oxidative stress. Heart Rhythm. 9:2023-2031.

45. Beretta-Piccoli C, Weidmann P, Schiffl H, Cottier C, Reubi FC. Enhanced cardiovascular pressor reactivity to norepinephrine in mild renal parenchymal disease. Kidney Int. 1982;22:297-303.

46. Konecny T, Park JY, Somers KR, et al. Relation of chronic obstructive pulmonary disease to atrial and ventricular arrhythmias. Am J Cardiol. 2014; $114: 272-277$

47. Hubatsch M, Kikeli P, Preg Z, et al. Risk factor for complex chronic comorbidities, a retrospective case-control study. Acta Medica Marisiensis. 2011;57:209-213.

48. Johnson LS, Juhlin T, Engström G, Nilsson PM. Reduced forced expiratory volume is associated with increased incidence of atrial fibrillation: the Malmo Preventive Project. Europace. 2014;16:182-188.

49. Acar $G$, Kahraman $H$, Akkoyun $M$, et al. Evaluation of atrial electromechanical delay and its relationship to inflammation and oxidative stress in patients with chronic obstructive pulmonary disease. Echocardiography. 2014;31:579-585.

50. Gan WQ, Man SF, Senthilselvan A, Sin DD. Association between chronic obstructive pulmonary disease and systemic inflammation: a systematic review and a meta-analysis. Thorax. 2004;59:574-580.

51. Heeringa J, Kors JA, Hofman A, van Rooij FJ, Witteman JC. Cigarette smoking and risk of atrial fibrillation: the Rotterdam Study. Am Heart J. 2008;156:1163-1169.

52. Ambrose JA, Barua RS. The pathophysiology of cigarette smoking and cardiovascular disease: An update. J Am Coll Cardiol. 2004;43:17311737.

53. Shan $\mathrm{H}$, Zhang $\mathrm{Y}$, Lu Y, et al. Downregulation of miR-133 and miR-590 contributes to nicotine-induced atrial remodelling in canines. Cardiovasc Res. 2009;83:465-472.

54. Laustiola KE, Kotamaki M, Lassila R, Kallioniemi OP, Manninen V. Cigarette smoking alters sympathoadrenal regulation by decreasing the density of beta 2- adrenoceptors. A study of monitored smoking cessation. J Cardiovasc Pharmacol. 1991;17:923-928.

55. Wang H, Yang B, Zhang L, Xu D, Wang Z. Direct block of inward rectifier potassium channels by nicotine. Toxicol Appl Pharmacol. 2000;164:97101.

56. Gallagher C, Hendriks JML, Elliott AD, et al. Alcohol and incident atrial fibrillation - A systematic review and meta-analysis. Int J Cardiol. 2017;246:46-52.

57. Gallardo-Carpentier A, Aileru AA, Carpentier RG. Arrhythmogenic and antiarrhythmic actions of substances of abuse: effects on triggered activity. J Electrocardiol. 1997;30:137-142.

58. Qiao Y, Shi R, Hou B, et al. Impact of alcohol consumption on substrate remodeling and ablation outcome of paroxysmal atrial fibrillation. J Am Heart Assoc. 2015;4:e002349.

59. Laszlo R, Eick C, Schwiebert M, et al. Alcohol-induced electrical remodeling: effects of sustained short-term ethanol infusion on ion currents in rabbit atrium. Alcohol Clin Exp Res. 2009;33:1697-1703.

60. Gould L, Reddy CV, Becker W, On KC, Kim SG. Electrophysiologic properties of alcohol in man. J Electrocardiol. 1978;11:219-226.

61. Cardy MA, Donnerstein RL, Kelly LF, Bittner NH, Palombo GM, Goldberg SJ. Acute effects of ethanol ingestion on signal-averaged electrocardiograms. Am J Cardiol. 1996;77:1356-1357.

62. Süfke $\mathrm{S}$, Fiedler $\mathrm{S}$, Djonlagic $\mathrm{H}$, Kibbel T. Continuous analysis of heart rate variability for examination of cardiac autonomic nervous system after alcohol intoxication. Med Klin (Munich.) 2009;104:511-519.

63. Shao Q, Liu T, Korantzopoulos P, Zhang Z, Zhao J, Li G. Association between air pollution and development of atrial fibrillation: A metaanalysis of observational studies. Heart Lung. 2016;45:557-562.

64. Peters $A$, Fröhlich $M$, Döring $A$, et al. Particulate air pollution is associated with an acute phase response in men; results from the 
MONICA-Augsburg Study. Eur Heart J. 2001;22:1198-1204.

65. Schwartz J, Litonjua $A$, Suh $\mathrm{H}$, et al. Traffic related pollution and heart rate variability in a panel of elderly subjects. Thorax. 2005;60:455-461.

66. Liao D, Shaffer ML, He F, et al. Fine particulate air pollution is associated with higher vulnerability to atrial fibrillation-the APACR study. J Toxicol
Environ Health A. 2011;74:693-705

67. Rienstra M, Hobbelt AH, Alings $M$, et al. Targeted therapy of underlying conditions improves sinus rhythm maintenance in patients with persistent atrial fibrillation: results of the RACE 3 trial. Eur Heart $\mathrm{J}$. 2018;39:2987-2996. 